\title{
Chronic Pyriform Sinus-Pleural-Mediastinal Fistula Secondary to an Internal Penetrating Trauma by a Toothbrush
}

\author{
Qing Cheng, Banghua Liu*, Jibao Wang \\ Department of Otorhinolaryngology, Union Hospital, Tongji Medical College, Huazhong University of Science \\ and Technology, Wuhan, China \\ Email: ${ }^{*}$ liubanghua2010@163.com
}

Received 29 January 2014; revised 18 February 2014; accepted 13 March 2014

Copyright (C) 2014 by authors and Scientific Research Publishing Inc.

This work is licensed under the Creative Commons Attribution International License (CC BY). http://creativecommons.org/licenses/by/4.0/

(c) (i) Open Access

\begin{abstract}
Objective: To report an unusual case of pyriform sinus-pleural-mediastinal fistula secondary to internal penetrating trauma by a toothbrush, with emphasis on management options. Case report: A 26-year-old woman swallowed a toothbrush in a violent manner 8 months ago in an attempt of self-mutilation. She immediately complained of neck pain and dysphagia. She was diagnosed with pyriform sinus-pleural-mediastinal fistula. The fistula was repaired. Primary closure was performed and covered with the sternocleidomastoid muscle flap, and reinforced with thyroid tissue and surrounding tissues. The patient did not present any complications or recurrence, and she gained weight after the operation. Conclusion: Early recognition of hypopharyngeal perforation is important, and in selected cases, immediate repair of the perforation can lead to a good prognosis.
\end{abstract}

\section{Keywords}

Hypopharynx; Esophageal Perforation; Penetrating Wounds

\section{Introduction}

Perforation of the hypopharynx is a rare injury, accounting for less than $2 \%$ of all perforations in the pharynxgoesophageal region [1]. The most common cause of hyperpharyngeal perforation is iatrogenic. Delays in diagnosis and treatment may lead to serious complications and a high mortality rate. The current treatment of hypopharyngeal perforation includes conservative treatment and surgical intervention. Pyriform sinus perforations with a chronic disease process are even rarer. To date, a toothbrush has not been reported as the cause of an in-

\footnotetext{
${ }^{*}$ Corresponding author.
}

How to cite this paper: Cheng, Q., Liu, B.H. and Wang, J.B. (2014) Chronic Pyriform Sinus-Pleural-Mediastinal Fistula Secondary to an Internal Penetrating Trauma by a Toothbrush. International Journal of Otolaryngology and Head \& Neck Surgery, 3, 95-100. http://dx.doi.org/10.4236/ijohns.2014.32019 
ternal penetrating injury to the hypopharyngeal region. We present a clinical case of pyriform sinus-pleural-mediastinal fistula, which to our knowledge, has not been previously described. Clinical presentation, diagnostic imaging evaluation, and operative management of the patient are described, as well as a review of the literature.

\section{Case Report}

A 26-year-old woman swallowed a toothbrush in a violent manner 8 months ago while attempting self-mutilation. She immediately complained of neck pain and dysphagia. Conservative treatment was applied without any improvement in the local hospital. The patient was then treated with debridement and drainage of a neck abscess, mediastinal abscess, and thoracic close drainage, as well as repair of pyriform sinus perforation with the greater omentum 7 months ago. Jejunostomy was also performed at the same time. However, pyriform sinus perforation recurred 1 week later. A hypopharynx stent implantation was then performed, but the patient forced the stent out by herself. The patient was then transferred to our hospital and admitted with the diagnosis of pyriform sinuspleural-mediastinal-fistula and empyema.

A physical examination showed that her temperature was $36.5^{\circ} \mathrm{C}$, her pulse was $76 / \mathrm{min}$, respiration was 20/min, and blood pressure was 97/61 mmHg. The patient exhibited cachexia and an anemic appearance. The right side of the thorax was slightly collapsed with an external thoracic drainage tube and weak breathing sounds. A nutrient canal was placed in the abdomen. A routine blood test showed the following: white blood cell count, $7.9 \mathrm{G} / \mathrm{L}$; neutrophilic granulocytes, $60.25 \%$; and $\mathrm{Hb}, 6.9 \mathrm{~g} / \mathrm{L}$. An electrocardiogram showed sinus tachycardia $(116 / \mathrm{min})$ with right axis deviation. Chest X-ray films and visualization of the esophagus with iodized oil showed right pleural thickening with adhesions, encapsulation, and pneumatosis in the chest wall, and pyriform sinus-pleural-mediastinal fistula (Figure 1).

Under gastroscopy, we found a fistula opening at the bottom of the right pyriform sinus, below the level of the arytenoid cartilage and close to the esophageal entrance (Figure 2), which was diagnosed as hypopharynx fistulization. Computed tomography (CT) of the neck and chest showed that the hypopharynx and esophagus could not be clearly seen with a nodular dense shadow. The right pleura was thickened, showing postoperative

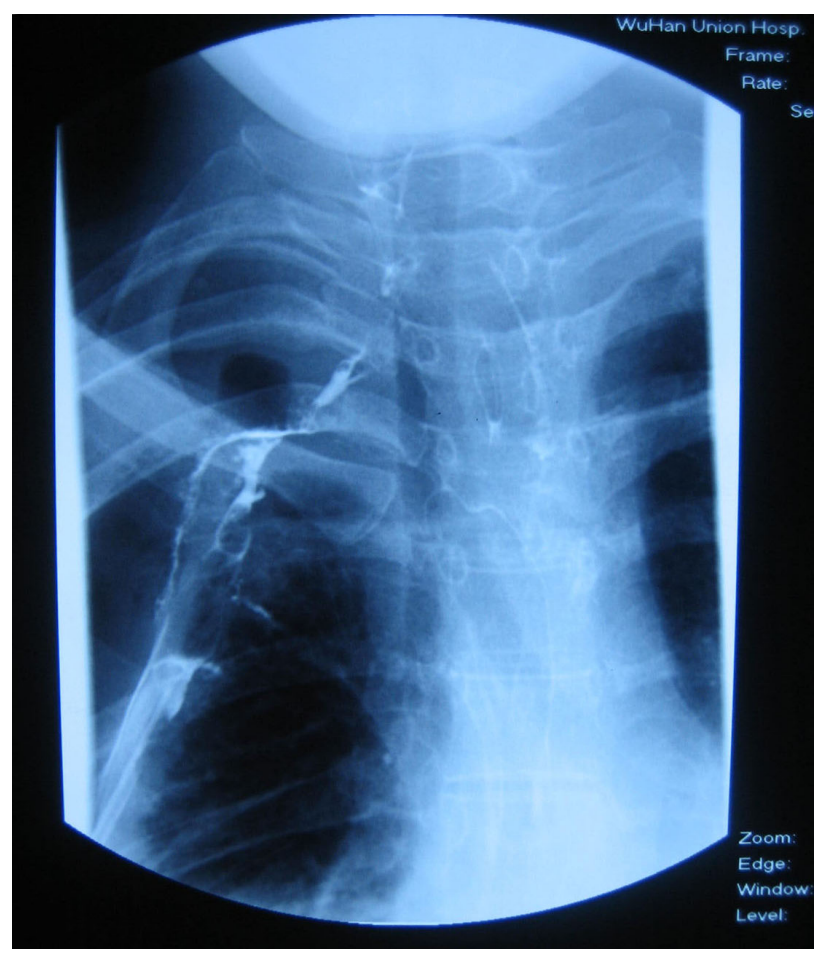

Figure 1. Water-soluble contrast agent (iodized oil) can be seen leaking along the right side of the pleura and thoracic drainage tube through a pyriform sinus fistula. 
changes of chest drainage, accompanied by collapse of the right thorax. Local consolidations in the superior and inferior lobes of the right lung were observed (Figure 3). Magnetic resonance imaging showed abnormal signals in the hypopharynx, right thorax, thoracic wall, and right lung, indicating pyriform sinus-pleural-mediastinal fistula accompanied by infection of the right lung and thoracic wall.

\section{Treatment}

The patient was given surgical treatment. The neck was opened with an anterior incision parallel to the sternocleidomastoid muscle, and the pharynx and esophagus were mobilized from the prevertebral fascia. The fistula was repaired. Primary closure was performed, and covered with the sternocleidomastoid muscle flap and reinforced

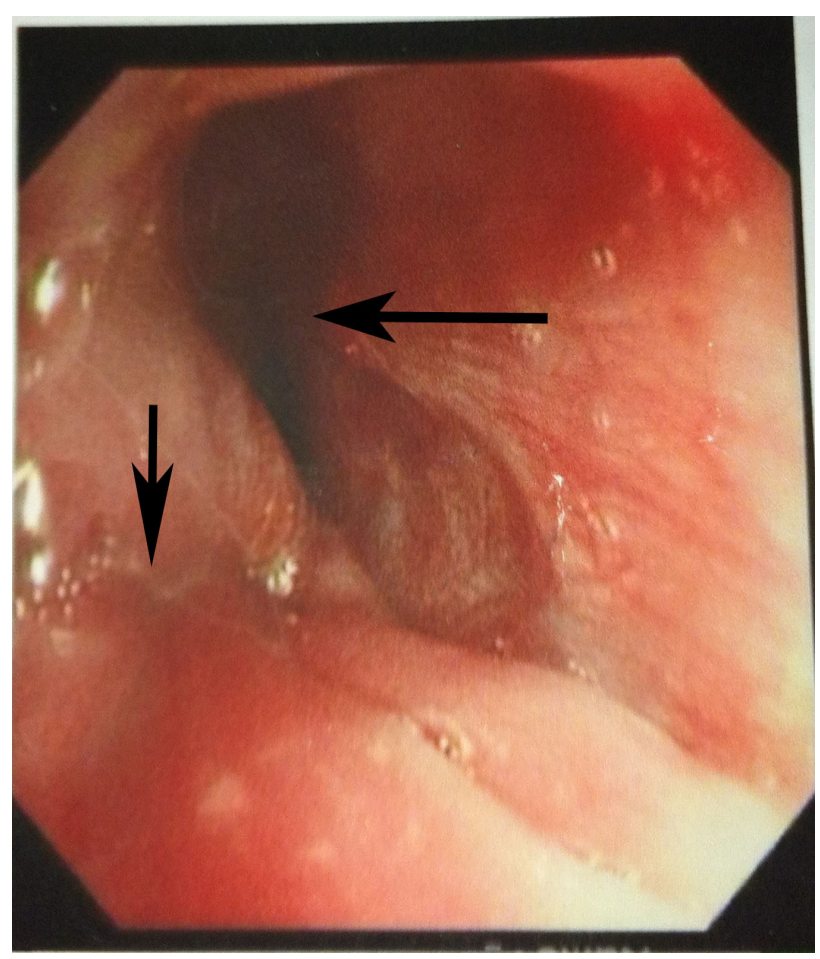

Figure 2. Under gastroscopy, a fistula (long arrow) is shown at the bottom of the right pyriform sinus, below the level of the arytenoid cartilage, close to the esophageal inlet (short arrow).

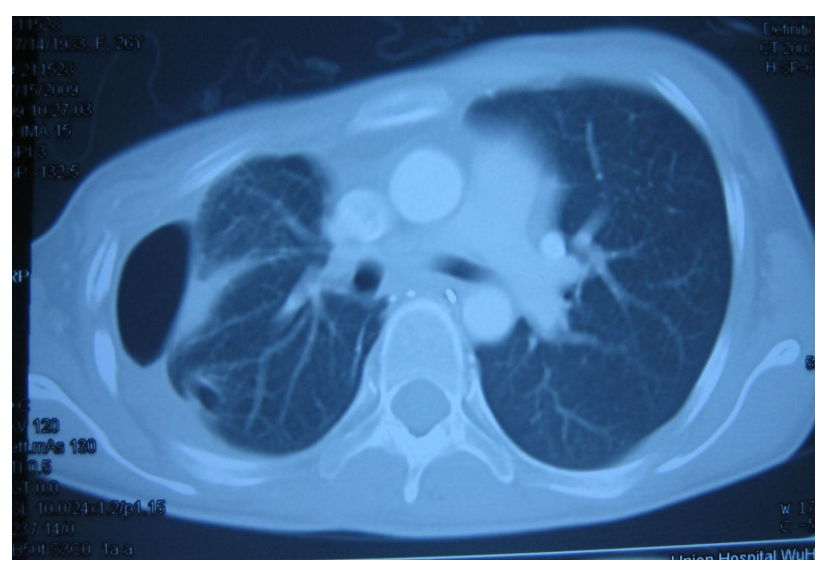

Figure 3. CT demonstrating right pleural thickening, collapse of the right side of the thorax, and local consolidations in the superior and inferior lobes of the right lung. 
with thyroid tissue and surrounding tissue. A nasogastric tube was placed and a drainage tube was inserted into the incision. Postoperatively, the patient was given intravenous antibiotics and best support care. The jejunal feeding tube was removed within 24 hours after the operation and replaced by nil per os for 10 days. After 48 hours, the cervical drainage tube was removed without drains from the tube. The patient began to receive a semi-liquid diet orally on the $10^{\text {th }}$ day after the operation, and the thoracic drainage tube was removed at the same time. On the $14^{\text {th }}$ day after the operation, the nasogastric tube was also removed and visualization of the esophagus with iodized oil was performed again. This showed contrast agent extravasation from both sides of the pyriform sinus into the trachea without leakage, which indicated intact hypopharyngeal repair (Figure 4). Twenty-one days after the operation, visualization of the esophagus with iodized oil showed extravasation of contrast agent from the epiglottis to the endotrachea, whereas the esophagus showed no significant stenosis or filling defects, suggesting swallowing dysfunction. The patient progressively received a regular diet, occasionally regurgitating when eating. She was then discharged home and was followed up. Her esophagus was reexamined using iodized oil 6 months after the operation. There was no extravasation of contrast agent and the esophagus showed no significant stenosis or filling defects. Currently, more than 3 years after being discharged from hospital, she has not presented with any complications or recurrence, and has gained weight.

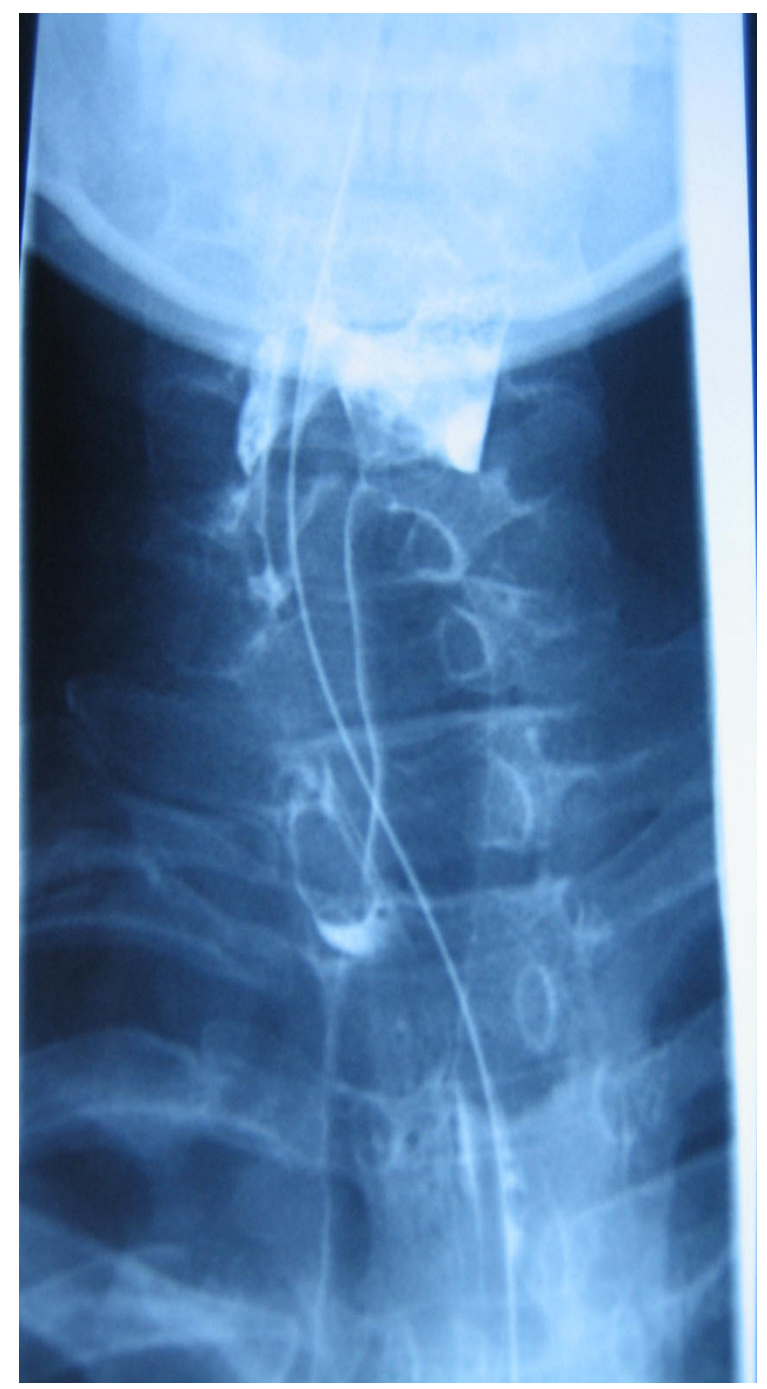

Figure 4. Extravasation of contrast agent from both sides of the pyriform sinus into the trachea without leakage can be seen, demonstrating an intact hypopharyngeal repair (2 weeks after operation). 


\section{Discussion}

The most common sites of hypopharyngeal perforation are the weak posterior hypopharyngeal wall and pyriform sinus. The pyriform sinus is well covered with muscle, which is comparatively resistant to penetration because of its strong extensibility [2]. Therefore, the violent impact of a toothbrush in our case led to internal penetrating injury to the pyriform sinus. Foreign bodies, blunt or penetrating neck wounds, iatrogenic or spontaneous causes, or malignant diseases cause pyriform sinus perforation, among which the iatrogenic cause is the most common. Unclear anatomy, lack of clinical experience, and a blind violent operation easily lead to injury or perforation of the pyriform sinus, pharynx, or esophagus. Transoral injuries, such as sword swallowers, knitting needles, endoscopy, and foreign bodies have been previously described [3]-[5]. However, injury of the pyriform sinus, pharynx or esophagus caused by penetration of a toothbrush has not been previously reported.

Once pyriform sinus perforation occurs, symptoms in the early stage may be mild, such as neck pain, limitation of cervical motion, odynophagia, hoarseness, dyspnea, subcutaneous emphysema, and mediastinal emphysema. Later clinical manifestations with fever and local swelling may be indicative of retropharyngeal or parapharyngeal abscess or severe complications, such as mediastinitis, neck abscess or fistula, and pyriform sinuspleural-mediastinal fistula, where the prognosis is directly related to delays in diagnosis or management [6].

Rapid diagnostic tests are required for suspected perforation, and an X-ray should be performed first. X-rays often reveal signs of subcutaneous or mediastinal emphysema, as well as pleural effusion. However, the results from plain X-rays are sometimes negative, which is insufficient to rule out perforation. In this case, contrast $\mathrm{X}$-ray studies provide confirmation of perforation and information about its location and extent. In some cases, visualization of the esophagus with iodized oil may lead to missed diagnosis of perforation with a false negative rate of $25 \%-50 \%$. If the diagnosis cannot be confirmed using contrast X-ray, an esophageal barium meal should be performed because of its higher sensitivity, especially in small tears or perforations [7]. This test may be hazardous when there is extravasation, and therefore, clinical application should be cautious. Contrast X-ray tests still have a negative rate of $10 \%$. Therefore, comprehensive analysis concerning clinical manifestations is essential. Endoscopic examination can directly show perforation, but some small tears or perforation could still be missed. High-resolution CT scans provide important diagnostic imaging data of pyriform sinus perforation, such as its precise localization and size. Furthermore, CT differentiates retropharyngeal cellulitis and abscesses and shows foreign bodies. Extraluminal air, fluid, and esophageal thickening are considered characteristic signs of perforation. High-resolution CT scans may be helpful in cases in which clinical suspicion of perforation remains despite negative contrast imaging results.

The treatment of pyriform sinus perforation remains controversial. Physicians should be aware of this type of lesion, and the diagnosis should be confirmed and the patient properly treated as early as possible. Treatment, including conservative treatment and surgical intervention, depends on the situation of perforation, such as the size of injury, cause, site, complications, and patient's age and physical condition. Conservative treatment of pyriform sinus perforation consists of nil per os, intravenous broad-spectrum antibiotics, and either gastric or parenteral nutrition. Surgical intervention includes neck drainage, chest or upper mediastinal drainage, and transoral endoscopic or surgery repair. Currently, it is recommended that conservative treatment is suitable for the following patients suffering from pyriform sinus perforation [2]-[5]: 1) patients whose perforation is quickly diagnosed and lasts less than 12 hours; 2) patients with a small perforation $(<2 \mathrm{~cm})$ located in the cervical esophagus or hypopharynx; 3) patients without symptoms or with mild symptoms and a lack of complications; 4) patients taken nothing orally since the injury; and 5) patients whose condition could not tolerate an operation. A large perforation ( $>2 \mathrm{~cm}$ ) lasting more than 12 hours or any complications require exploration and repair by operation if possible and adequate drainage. However, successful conservative treatment of large perforations up to $5 \mathrm{~cm}$ long has been reported [8]. Therefore, close observation is necessary during conservative treatment, and surgical intervention should be followed without delay if the patient's condition deteriorates.

\section{Conclusion}

Pyriform sinus perforation is a type of hypopharyngeal perforation. In our case, perforation caused by a toothbrush accompanied by pyriform sinus-pleural-mediastinal fistula is an extremely rare injury. Early diagnosis is particularly important. The therapeutic options depend on the complications and the patient's condition. Close observation is also essential. Mortality and prognosis are directly related to delays in diagnosis and treatment. 


\section{Summary}

1) Hypopharyngoesophageal perforation develops rapidly, easily leading to serious complications. If early conservative treatment fails, surgical repair should be followed as soon as possible.

2) The key to treatment of this lesion is to be aware of its possibility, as well as to confirm the diagnosis and treat the patient properly as early as possible.

3) Simple sutures are difficult to heal because of insufficient blood supply to the hypopharynx. Muscle flap coverage allows an increase in local blood supply, enhancing immunity and reducing friction and compression of surrounding tissue. The sternocleidomastoid is recommended for closure of hypopharyngoesophageal perforations, which was applied in our patient to repair pyriform sinus perforation. The muscle flap needs to be snugly placed.

4) Enhancement of blood supply to the surrounding tissue is important. In our patient, the repair was reinforced with thyroid tissue and surrounding tissue.

\section{References}

[1] Berry, B.E. and Ochsner, J.L. (1973) Perforation of the Esophagus: A 30 Year Review. The Journal of Thoracic and Cardiovascular Surgery, 65, 1-7.

[2] Stanley Jr., R.B., Armstrong, W.B., Fetterman, B.L., et al. (1997) Management of External Penetrating Injuries into Hypopharyngeal-Cervical Esopbageal Funnel. Journal of Trauma, 42, 675-679. http://dx.doi.org/10.1097/00005373-199704000-00016

[3] Moberly, A.C., Fritsch, M.H. and Mosier, K.M. (2011) Management of Sword-Swallower Injuries. The Journal of Laryngology \& Otology, 125, 217-219. http://dx.doi.org/10.1017/S002221511000215X

[4] Moriwaki, Y., Arata, S. and Iwashita, M. (2011) A Case of Cervical Esophageal Perforation after Transnasal Gastrointestinal Fibroscopy Using a Small-Caliber Fiber. Endoscopy, 43, E147-E148. http://dx.doi.org/10.1055/s-0030-1256211

[5] Choudhary, A.M., Roberts, I. and Gupta, T. (1999) Hypopharyngeal Perforation from a Swallowed Fork: A Brief Report and Comment. Connecticut Medicine, 63, 539-540.

[6] Engum, S.A., Grosfeld, J.L., West, K.W., et al. (1996) Improved Survival in Children with Esophageal Perforation. Archives of Surgery, 131, 604-610. http://dx.doi.org/10.1001/archsurg.1996.01430180030005

[7] Foley, M.J., Ghahremani, G.G. and Rogers, L.F. (1982) Reappraisal of Contrast Media Used to Detect Upper Gastrointestinal Perforations. Radiology, 144, 231-237.

[8] Christey, G.R. (2005) Blunt Handlebar Injury Causing Pharyngeal Perforation. Injury Extra, 36, 373-375. http://dx.doi.org/10.1016/j.injury.2004.11.024 